\title{
Study of structural, optical and mechanical properties of TiN and TiCN thin films
}

\author{
Omveer Singh $^{1 *}$, Raj P. Dahiya ${ }^{1,2}$ and Hitendra K. Malik ${ }^{3}$ \\ ${ }^{1}$ Centre for Energy Studies, Indian Institute of Technology Delhi, New Delhi - 110016 \\ ${ }^{2}$ Deenbandhu Chhotu Ram University of Science and Technology, Murthal -131039 \\ ${ }^{3}$ Department of Physics, Indian Institute of Technology Delhi, New Delhi - 110016 \\ *Email: poonia.omveer@gmail.com
}

The primary emphasis of the research on nanocrystalline TiN and TiCN thin films is tuning the desirable properties of these materials for our use, i.e. tuning of hardness which makes them ideal candidates for wear resistant applications. This all resulted in considerable advances in the areas of tribology like dry cutting and metal forming, mechanical deformation behaviour and electrical transport properties of TiN and TiCN thin films [1,2]. In the present work, Ti thin films of about $200 \mathrm{~nm}$ thickness were grown on Si substrate by electron beam evaporation method. Further, these films were nitrided by low energy ion beam implantation technique. A beam of nitrogen ions with fixed ion current $1 \mu \mathrm{A}$, energy $70 \mathrm{KeV}$ and $1 \times 10^{16}$ ions $/ \mathrm{cm}^{2}$ fluence was used in this process. After this one of the titanium nitride thin film was carbide with carbon ions at $1 \times 10^{15}$ ions $/ \mathrm{cm}^{2}$ fluence.

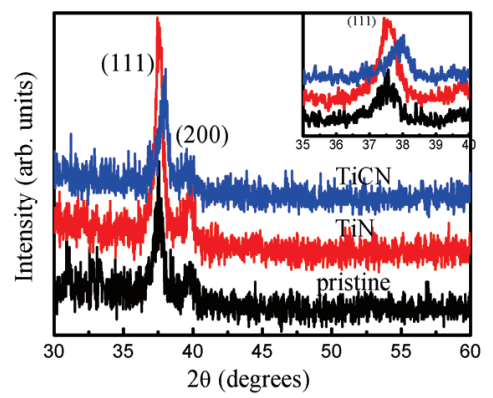

Figure 1: XRD pattern of pristine (Ti), TiN and TiCN

The XRD result of TiN film shows the shift in the (111) diffraction peak towards higher diffraction angle which demonstrate the strain in the film (Figure 1). Further shift was observed for TiN film after carbidation. AFM results confirm that the particle size $(\sim 20.65 \mathrm{~nm}, \sim 226$ $\mathrm{nm}$ and $\sim 124.73 \mathrm{~nm})$ and roughness $(\sim 28.2 \mathrm{~nm}$, $\sim 234 \mathrm{~nm}$ and $\sim 133 \mathrm{~nm}$ ) varies for pristine, TiN and TiCN films simultaneously (Figure 2). The vertical force-displacement curves of pristine (Ti), TiN and TiCN have been shown in Figure
3. The band gap diminution from $3.09 \mathrm{eV}$ to 2.13 $\mathrm{eV}$ was found for pristine to TiCN film which leads to enhancement in optical property. The hardness of $\sim 1.31 \mathrm{GPa}, \sim 1.33 \mathrm{GPa}$ and $\sim 1.35$ $\mathrm{GPa}$ was measured for pristine, $\mathrm{TiN}$ and $\mathrm{TiCN}$ thin films.

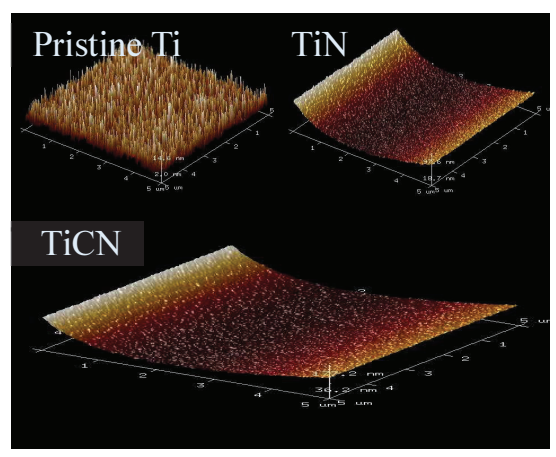

Figure 2: AFM images of pristine (Ti), TiN and TiCN

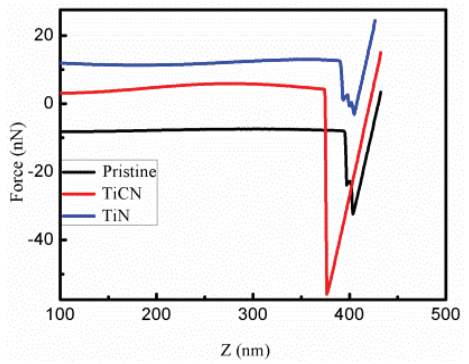

Figure 3: Vertical force-displacement curves of pristine (Ti), TiN and TiCN

Hence, the TiN films exhibit high hardness as compared to pure titanium but TiCN films have higher hardness in comparsion to both $\mathrm{Ti}$ and TiN. Other properties are also good such as structural, morphologcal and optical.

\section{References}

1. P. Le Clair, G. P. Berera and J. S. Moodera, Thin Solid Films 376 (2000) 9.

2. J. H. Kang and K. J. Kim, J. Appl. Phys. 86 (1999) 346. 